\title{
Examination of a Practical Aerobraking Guidance Algorithm
}

\author{
Steven W. Evans* and Greg A. Dukeman ${ }^{\dagger}$ \\ NASA Marshall Space Flight Center, Alabama 35812
}

\begin{abstract}
A practical real-time guidance algorithm has been developed for aerobraking vehicles that minimizes the pastaeropass $\Delta V$ requirement for orbit insertion while nearly minimizing the maximum heating rate and the maximum structural loads. The algorithm is general in the sense that a minimum of assumptions is made, thus greatly reducing the number of parameters that must be determined prior to a given mission. An interesting feature is that in-plane guidance performance is tuned by adjusting one mission-dependent parameter, the bank margin; similarly, the out-of-plane guidance performance is tuned by adjusting a plane controller time constant. Other features of the algorithm are simplicity, efficiency, and case of use. The algorithm is designed for, but not necessary restricted to, a trimmed vehicle with bank angle modulation as the method of trajectory control. Performance of this guidance algorithm during flight in Earth's atmosphere is examined by its use in an aerobraking testbed program. The performance inquiry extends to a wide range of entry speeds covering a number of potential mission applications. Favorable results have been obtained with a minimum of development effort, and directions for improvement of performance are indicated.
\end{abstract}

\section{Introduction}

$T \mathrm{~N}$ previous years proposed aerobraking guidance concepts ${ }^{1}$ were developed with computational efficiency as one of the major design goals. This was a carryover from a period when computing capability was a fraction of what it is today. To accommodate this limited capability, simplifying assumptions were made in order to avoid numerical integration of the equations of motion. As a result, the guidance algorithms were not very general or adaptable for future aerobraking mission planning, and laborious premission analyses were required to determine appropriate values for mission-dependent parameters needed because of the simplifying assumptions. The speed of today's computers permits more general real-time guidance modeling.

Numerous aerobraking guidance strategies have been developed over the years. Fuhry ${ }^{2}$ describes a guidance algorithm based on the numerical predictor-corrector of Gamble et al. ${ }^{1}$ Fuhry's ${ }^{2}$ exit phase logic for apoapsis control is similar to the present in that the guidance algorithm determines the constant bank angle trajectory that takes the current vehicle state to the desired exit conditions. Shipley and Ward ${ }^{3}$ discuss control strategies for aerobraking at Mars, providing a good discussion of issues relating to the transition between entry and exit phases of flight; the present algorithm addresses this transition in a novel way. Willcockson ${ }^{4}$ discusses the use of a numerical predictor-corrector algorithm that uses bank rate as a control variable for a continuously rolling vehicle, an approach not considered here. Braun et al..$^{5}$ discuss the use of a numerical predictor-corrector that uses a load relief strategy to explicitly limit heating rate during the entry phase. The present algorithm implicitly controls heating rate by issuing full lift-up commands (0 deg or 180 deg bank angle commands, as appropriate for the vehicle) until the guidance logic determines that continuing to fly lift up may result in a premature skip-out.

The algorithm described in this paper has been used in an aerobraking simulation testbed program that allows the introduction of variations from nominal values of atmospheric density, vehicle mass and aerodynamic parameters, and on-board navigation hardware outputs. The initial conditions and target orbit can also be spec-

Received June 26, 1993; revision received April 5, 1994; accepted for publication July 5, 1994. Copyright (C) 1994 by the American Institute of Aeronautics and Astronautics, Inc. No copyright is asserted in the United States under Title 17, U.S. Code. The U.S. Government has a royalty-free license to exercise all rights under the copyright claimed herein for Governmental purposes. All other rights are reserved by the copyright owner.

*Aerospace Engineer, Flight Mechanics Branch, Mail Stop EL58. Member AIAA.

${ }^{\dagger}$ Aerospace Engineer, Flight Mechanics Branch, Mail Stop EL58. ified as desired. The results of many simulations conducted with this program have been reduced to a set of entry corridors depicted on the entry speed-entry flight-path angle plane. These corridors indicate the capabilities of the algorithm and suggest ways in which the two tunable parameters should be adjusted to obtain desired results.

The paper is organized as follows. First an apoapsis controller is described. The strategy is to use constant bank angle trajectories to attain the desired apoapsis. Next a strategy is discussed that results in minimum $\Delta V$ and near-minimum peak heating rates and structural loading. The plane controller is then discussed. The orbit plane upon exit (inclination and longitude of ascending node) is implicitly controlled via the out-of-plane velocity. Finally, numerical results are presented in terms of entry corridors.

\section{Basic Apoapsis Controller}

The guidance algorithm examined in this paper is named the Generalized Reusable Guidance for Optimal Aerobraking (GREGOAR) scheme. In this algorithm, a Newton-Raphson approach is used to determine the constant bank angle trajectory that will attain the desired apoapsis. Although the equations of motion are numerically integrated, for simplicity they are reduced to the following set of planar equations of motion ${ }^{6}$ for a nonrotating, spherical planet:

$$
\begin{gathered}
\dot{r}=v \sin \gamma \\
\dot{v}=-D_{a}-g \sin \gamma \\
\dot{\gamma}=L_{a} \cos \phi / v+(v / r-g / v) \cos \gamma
\end{gathered}
$$

Here $r$ is vehicle position magnitude, $v$ is inertial velocity magnitude, $\gamma$ is the inertial flight-path angle, $\phi$ is the bank angle, $L_{a}$ and $D_{a}$ are the aerodynamic lift and drag accelerations given by

$$
L_{a}=\frac{1}{2} \rho v_{r}^{2} S C_{L} / m \quad D_{a}=\frac{1}{2} \rho v_{r}^{2} S C_{D} / m
$$

and $g$ is the gravitational acceleration given by

$$
g=\mu / r^{2}
$$

In order to have adaptability with respect to variations in atmospheric density and with respect to uncertainties in the vehicle's aerodynamic coefficients and mass, estimates of these quantities must be made during the aeropass. We assume that accelerometer measurements are available, so that measured lift and drag acceleration magnitudes can be computed from the sensed acceleration. 


\section{Examination of a Practical Aerobraking Guidance Algorithm S. W. Evans and G. A. Dukeman}

Reprinted from

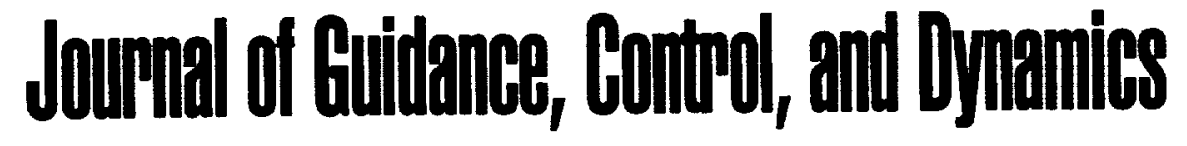

Volume 18, Number 3 , Pages 471-477

\section{CAIAA.}

A publication of the

American Institute of Aeronautics and Astronautics, Inc.

370 L'Enfant Promenade, SW

Washington, DC 20024-2518 
Then, following Gamble et al., ${ }^{1}$ the composite quantities $\rho C_{L} / m$ and $\rho C_{D} / m$ can be estimated by

$$
\begin{aligned}
& \left(\frac{\rho C_{L}}{m}\right)_{\text {est }}=\frac{2 L_{a, \text { mess }}}{v_{r}^{2} S} \\
& \left(\frac{\rho C_{D}}{m}\right)_{\text {est }}=\frac{2 D_{a, \text { meas }}}{v_{r}^{2} S}
\end{aligned}
$$

The estimates for each guidance cycle are input to a low-pass filter to obtain the values used in the guidance computations.

The relative velocity $v_{r}$ in Eq. (2) can be computed by assuming the difference between inertial and relative velocity magnitudes is a constant throughout the trajectory. This is necessary because of the use of planar equations of motion. Assuming flight is hypersonic and that aerodynamic forces are significant over a small altitude range, the lift and drag coefficients can be modeled as constants, again for simplicity.

The problem of determining the required bank angle $\phi$ is mathematically that of determining the zero of the nonlinear constraint function

$$
\left.\Psi(\phi)\right|_{r=r_{e x}}=r_{a_{t}}-\left.r_{a}(r, v, \gamma)\right|_{r=r_{e x}}
$$

where $r_{e x}$ is the radius at atmospheric exit, $r_{a_{l}}$ is the target radius of apoapsis, and $r_{a}$ is the actual radius of apoapsis at the simulated exit from the atmosphere. There are a number of techniques available for solving $\mathrm{Eq}$. (5); the method used here is based on Newton's method, with the Jacobian computed using a variational approach. To decrease the nonlinearity of the problem, the solution of Eq. (5) is obtained by iterating on the cosine of bank angle instead of on bank angle directly. If the solution for a given guidance cycle happens to result in $|\cos (\phi)|>1$, which is referred to as guidance saturation, then the bank angle command is limited to 0 deg or 180 deg as appropriate. For the next guidance cycle the limited solution from the previous guidance cycle is used as the initial guess.

The set of variational equations used to solve Eq. (5) is developed here. The Newton-Raphson iteration from the cosine of required bank, $c \phi$, is written

$$
c \phi_{i+1}=c \phi_{i}-\left.\frac{\Psi}{\mathrm{d} \Psi / \mathrm{d}(c \phi)}\right|_{c \phi=c \phi_{i}}
$$

where $\Psi$ is the constraint function (5) and $\mathrm{d} \Psi / \mathrm{d}(c \phi)$ is the Jacobian, both of which are evaluated at atmospheric exit. Using conservation of energy and angular momentum, the constraint function can be expressed as

$$
\Psi=\Psi(r, v, \gamma)=1+2 \mu\left(1 / r_{a_{t}}-1 / r\right) / v^{2}-r^{2} \cos ^{2} \gamma / r_{a_{t}}^{2}
$$

The Jacobian in Eq. (6) is expressed via the chain rule as

$$
\frac{\mathrm{d} \Psi}{\mathrm{d}(c \phi)}=\frac{\mathrm{d} \Psi}{\mathrm{d} r} \frac{\partial r}{\partial \gamma} \frac{\mathrm{d} \gamma}{\mathrm{d}(c \phi)}+\frac{\partial \Psi}{\partial v} \frac{\partial v}{\partial \gamma} \frac{\mathrm{d} \gamma}{\mathrm{d}(c \phi)}+\frac{\partial \Psi}{\partial \gamma} \frac{\mathrm{d} \gamma}{\mathrm{d}(c \phi)}
$$

It is a routine matter to obtain analytical expressions for the partials of $\Psi$ with respect to $r, v$, and $\gamma$. The rest of the components needed in Eq. (8) must be obtained numerically as follows. Using the relation

$$
\frac{\mathrm{d}(\partial x(t) / \partial \alpha)}{\mathrm{d} t}=\frac{\partial(\mathrm{d} x(t) / \mathrm{d} t)}{\partial \alpha}
$$

variational equations governing the motion can be written as

$$
\begin{gathered}
\frac{\mathrm{d}(\partial r / \partial \gamma)}{\mathrm{d} t}=\frac{\partial(\mathrm{d} r / \mathrm{d} t)}{\partial \gamma}=\frac{\partial(v \sin \gamma)}{\partial \gamma}=\frac{\partial v}{\partial \gamma} \sin \gamma+v \cos \gamma \\
\frac{\mathrm{d}(\partial v / \partial \gamma)}{\mathrm{d} t}=-D_{a}\left(\frac{\partial \rho}{\partial r} \frac{\partial r / \partial \gamma}{\rho}+2 \frac{\partial v / \partial \gamma}{V_{r}}\right) \\
+2 \frac{g}{r} \frac{\partial r}{\partial \gamma} \sin \gamma-g \cos \gamma \\
\frac{\mathrm{d}(\partial \gamma / \partial(c \phi))}{\mathrm{d} t}=\frac{L_{a}}{v}
\end{gathered}
$$

with the initial conditions

$$
\begin{gathered}
\frac{\partial r}{\partial \gamma}\left(t=t_{0}\right)=0 \\
\frac{\partial v}{\partial \gamma}\left(t=t_{0}\right)=0 \\
\frac{\partial \gamma}{\partial(c \phi)}\left(t=t_{0}\right)=0
\end{gathered}
$$

Equations (10) and (11) constitute an initial-value problem in ordinary differential equations, which can be numerically integrated from the current time to atmospheric exit to give the required partials $\partial r / \partial \gamma, \partial v / \partial \gamma$, and $\partial \gamma / \partial(c \phi)$. In practice, the states $r, v, \gamma$ and the partials $\partial r / \partial \gamma, \partial v / \partial \gamma, \partial \gamma / \partial(c \phi)$ are propagated simultaneously to atmospheric exit, giving, in a single integration, all the information needed for use in Eq. (6). These equations are implemented in a noniterative mode; i.e., Eq. (6) is used only once per execution of the algorithm. The assumption used here is that the time between subsequent executions is small and therefore the vehicie state does not change significantly between executions. This eliminates the need for convergence criteria.

An adaptive step-size fifth-order Runge-Kutta-Fehlberg numerical integrator was chosen to perform the required integration because it is accurate and efficient and requires a relatively small amount of code and overhead. The integrated trajectories do not need to be computed very accurately because, due to navigation and modeling errors, the target can be attained with only limited accuracy anyway. Our experience in work on the aeroassist flight experiment (AFE) program has shown that $0.1 \mathrm{~km}$ in position error in the integration is adequate. Typically, no more than 15-20 integration steps are required to compute a simulated trajectory.

To ensure that the numerical integration process inside the guidance proceeds as intended, tests are performed after each integration step to detect simulated vehicles that are "trapped" in the atmosphere due to too much negative lift being modeled. If the (simulated) apoapsis is inside the sensible atmosphere or if the (simulated) minimum altitude is below some specified value, say $50 \mathrm{~km}$ for Earth or $30 \mathrm{~km}$ for Mars, then the numerical integration process is stopped. The amount of positive lift is then increased by incrementing the simulated cosine of bank by some specified amount, say \pm 0.1 , and the integration process is started anew from $i_{0}$.

The equations and methodology described in the next section constitute the guidance logic used during the exit or ascent phase of the aeropass. This guidance phase is initiated at an appropriate point in the aeropass as determined by the entry phase logic discussed in the following section.

\section{Optimal Apoapsis Controller}

Extensive aerobraking trajectory optimization work has been done in recent years. ${ }^{7-9}$ The strategy used here is suggested by the results of optimization work done by Miele and others, ${ }^{10,11}$ who have shown two important results:

1) If both atmospheric entry speed and flight-path angle are fixed, the optimal trajectory is a two-arc trajectory: an entry arc flown at full positive lift followed by an exit arc flown at full negative lift.

2) If only atmospheric entry speed is fixed, then the flight-path angle can be chosen so that the optimal trajectory is a one-arc trajectory flown at full negative lift.

The trajectory of result 2 is a special case of the trajectory of result 1 where the switch to full negative lift occurs at entry.

Here, "optimal" is used in the sense of minimum $\Delta V$ for postaeropass orbit insertion. ${ }^{3.12}$ Optimization results have shown that the trajectories discussed here are characterized by nearminimum values of peak heating rate and structural loading. ${ }^{12}$ The optimal trajectories at aeropass exit satisfy the apoapsis constraint (5).

Numerical computation of this class of optimal trajectories is simple (once the form is known), in that, in the case of result 1 , all that is needed is to iterate on a switching time (i.e., the time to switch from full positive lift to full negative lift) such that the vehicle attains the target apoapsis radius at exit. In the case of result 
2 , all that is needed is to determine the shallowest flight-path angle such that, at full negative lift, the vehicle attains the target apoapsis radius at exit.

In a real-time guidance environment, result 2 does not have much practical use since the guidance discussed here becomes active at atmospheric entry and so obviously cannot influence an entry fightpath angle already determined by the exoatmospheric trajectory. Rather, result 2 is suggestive of a premission or pre-entry optimization (to determine entry flight-path angle) that must be done in conjunction with a real-time guidance algorithm based on result 1. Thus the guidance strategy discussed here is derived with result 1 as a basis.

Result 1 cannot be used directly because the magnitude and effects of dispersions (atmospheric, commanded bank angle reversals, navigation errors, etc.) that will be encountered during the exit (negative-lift) phase are unknown. If result 1 is used directly and, for example, the encountered atmospheric density were less than the atmospheric model predicted, there would not be enough lift capability to prevent a premature skip-out. What is needed is some performance reserve to account for the dispersions that the vehicle will encountered during the exit phase. This reserve is obtained by introducing a "bank margin," a premission-determined positive bank angle increment, which is used during the entry phase guidance mode in the simulation of the exit (negative-lift) arc. The exit phase arc is simulated as a constant bank trajectory flown at full negative lift less the bank margin (hereafter referred to as quasi-full negative lift). This has the effect of causing the guidance logic to start the exit phase earlier than it really needs to. Once the exit phase guidance mode has been triggered, it solves for the bank angle required to hit the target apoapsis during each subsequent guidance cycle using the true vehicle lift capability (no bank margin included).

Instead of solving for the switching time from entry phase to exit phase, the entry phase guidance computes one trajectory per guidance cycle simulating flight using quasi-full negative lift; in the meantime, it commands full lift-up. When the simulation indicates that a skip-out will occur despite being at quasi-full negative lift, the exit phase guidance is initiated. This strategy allows the vehicle to fly lift up as long as possible, thus minimizing penetration into the atmosphere and consequently reducing heating rates and structural loads while avoiding skip-out. It also maximizes perigee altitude, and so minimizes the $\Delta V$ needed to raise perigee out of the atmosphere after the aeropass is completed. ${ }^{3}$

As bank margin is increased, the guidance algorithm triggers the exit phase guidance earlier in the trajectory. This fact can be used in cases where the higher values of total heating loads associated with these optimal trajectories are more of a concern than peak heating rates.

Note that all of the gains and many of the mission-dependent parameters so common in other guidance algorithms have been replaced in this optimal apoapsis controller by a single missiondependent parameter, the bank margin. This feature of the algorithm makes it readily "portable" (or "reusable") from one vehicle to another and from one set of entry conditions to another, even to the extent of changing planets at which the aeropass is to take place. As a contrary example, in the scheme of Gamble et al. ${ }^{1}$ gains on dynamic pressure and altitude rate must be adjusted for every change in entry conditions, target conditions, and/or vehicle parameters.

Another feature is that the time to transition from entry to exit phase is determined completely in real time: Prescribed trigger or transition velocities, which may have to be selected on the basis of trial and error, ${ }^{1.3}$ are not necessary. A computationally desirable feature is that in the entry phase only one integrated trajectory need be generated (without the variational trajectories), and furthermore, early in the aeropass these are shor-lived trajectories since the simulated vehicles become trapped in the atmosphere. The exit phase, which is inherently more central processing unit (CPU) intensive, does not start until the time to exit has been reduced somewhat in the entry phase.

\section{Real-Time Orbital Plane Controller}

The form of the plane controller is based on a first-order system where the state variable being controlled is the out-of-plane velocity component, that is, the vehicle's velocity component along a unit vector perpendicular to the desired orbit plane. Thus, if the magnitude of out-of-plane velocity is driven to a small value, the same is automatically done with the plane error. Starting with the homogeneous equation

$$
\frac{\mathrm{d} y}{\mathrm{~d} t}+\frac{y}{\tau}=0
$$

where $y$ is the current out-of-plane velocity (measured positive along the unit vector antiparallel to the desired unit angular momentum vector) and $\tau$ is a time constant, it is assumed that the out-of-plane lift component is the only out-of-plane force. Thus Eq. (12) may be written as

$$
L_{a} \sin \phi+y / \tau=0
$$

Solving for $\sin \phi$ and expanding $L_{a}$, we obtain

$$
\sin \phi=\frac{-2 m y}{\tau \rho V_{r}^{2} C_{L} S}
$$

The sign of $\sin \phi$ gives the appropriate sign of the bank command whereas the magnitude of $\sin \phi$ is a measure of the current plane error and thus can be used to determine when to switch the sign of the commanded bank angle. In practice, when the magnitude of $\sin \phi$ is larger than 1, the sign of the commanded bank angle is set to that of $\sin \phi$ :

$$
\operatorname{sgn}\left(\phi_{\mathrm{cmd}}\right)=\operatorname{sgn}(\sin \phi)
$$

This commanded bank sign is used in subsequent guidance cycles until $\sin \phi$ changes sign again and $|\sin \phi|$ becomes larger than 1 .

Inspection of Eq. (14) reveals that the magnitude of $\sin \phi$ becomes larger as dynamic pressure decreases. This results in increased sensitivity to plane error as atmospheric exit is approached and less sensitivity to plane error when dynamic pressure is large. This is precisely what is desired because larger plane errors deep in the atmosphere are acceptable; i.e., there is plenty of control authority available to correct for them; higher in the atmosphere less plane error is tolerated as exit is approached.

Note that satisfactory guidance performance can be achieved by adjusting $\tau$. Thus, as in the apoapsis controller case, we have a situation wherein only one critical parameter value needs to be determined prior to a mission. An important point is that large values of $\tau$ result in fewer roll reversals and large plane error at exit, whereas, on the other hand, small values of $\tau$ result in more roll reversals and small plane error at exit. Thus, it is a matter of trading off roll reversals for plane error.

\section{Numerical Testing}

A version of the GREGOAR guidance logic discussed above has been coded into a FORTRAN subroutine. The code accepts a variety of inputs, so that it may be used with different planets, vehicles, and entry conditions. A three-degree-of-freedom aerobraking guidance test bed program, the Aerobraking Orbital Transfer Technology (ABOTT) program, was used to examine the performance of GREGOAR. Inputs to ABOTT allow selection of vehicle mass, aerodynamic parameters, initial state vector, and other parameters necessary to conduct the simulation. In addition, multiple-run sets can be started with simulation initial conditions varied between runs, to allow statistical studies of the test algorithm to be performed.

$\mathrm{ABOTT}$ assumes that the only control variable is the vehicle bank angle. Although the guidance models assume instantaneous bank angle responses to commands, ABOTT models finite bank rates and accelerations, with a maximum bank rate of $20 \mathrm{deg} / \mathrm{s}$ and a maximum bank acceleration of $5 \mathrm{deg} / \mathrm{s}^{2}$ (the same values used by Shipley and $W^{2} d^{3}$ and in the AFE program ${ }^{13}$ ). Angle-of-attack variations about a trim value specific to the vehicle are simulated by allowing the lift and drag coefficients to fluctuate in a Gaussian manner from one aeropass to another, with mean and standard deviation of the Gaussian specified by the user. Sideslip is assumed to be a constant zero. Variations in vehicle mass from one run to another can also 
Table 1 Aeroassist fight experiment vehicle parameters

\begin{tabular}{lcccccc}
\hline \hline $\begin{array}{l}\text { Mass, } \\
\mathrm{kg}\end{array}$ & $\begin{array}{c}\text { Area, } \\
\mathrm{m}^{2}\end{array}$ & \multicolumn{2}{c}{ Drag coefficient } & & \multicolumn{2}{c}{ Lift coefficient } \\
\cline { 3 - 4 } & 14.314 & 1.3549 & 2.06717 & & -0.3807 & -0.09574 \\
\hline \hline
\end{tabular}

be applied. The mass is selected from a uniform distribution between user-specified maximum and minimum values containing the vehicle nominal value.

The program propagates the vehicle state from the initial time until either the vehicle exits the atmosphere or a preselected time limit is reached. At each time step within the propagation cycle the guidance algorithm is executed to compute the bank angle commands; auxiliary parameters such as atmospheric density, acceleration loads, aerodynamic heating, and dynamic pressure are calculated; and the equations of motion are numerically integrated using a fourth-order Runge-Kutta subroutine. When an aeropass simulation has been completed, summary data are output, including speed at entry, flight-path angle at entry, maximum aerodynamic load encountered, apogee altitude of the orbit at exit, and $\Delta V$ to circularize at the target altitude.

For the multirun cases used in this study, ABOTT computes dispersions to be applied to the vehicle mass, aerodynamic coefficients, and position and velocity vectors within the specified limits and selects an atmosphere model profile from a table specified by the user. Each aeropass simulation of a set is begun after the initial conditions have been modified in this way. When a sufficient number of runs have been made in the vicinity of a given initial point, i.e., a particular entry speed-entry flight-path angle combination, a step is made to the next initial point and a new set of runs is performed in its vicinity. Statistics for selected output parameters at each step are then compiled from the results.

The vehicle parameters used in this study are those for the AFE vehicle. This vehicle's design parameters are well known and form a convenient data set ${ }^{13}$; nominal values are given in Table 1 . The stated lift and drag coefficients hold for the vehicle flying at its trim angle of attack of $-17 \mathrm{deg}$. The combination of the AFE vehicle and GREGOAR guidance package will be referred to as the "study configuration."

In this study, atmospheric data are read from a table consisting of 101 cases generated by the Global Reference Atmosphere Model (GRAM) program. ${ }^{14}$ The tabular data include density, pressure, temperature, and wind speed and direction as functions of altitude. The data from case 1 are nominal values and the remaining 100 cases represent expected deviations about this nominal set. For a given run, ABOTT selects an atmospheric case index by using a random-number generator that chooses an integer from a uniform distribution between 1 and 101 .

\section{Entry Corridor Definition}

The simulations are initiated at an altitude of $122 \mathrm{~km}$ above Earth. The entry speed is varied between 9.0 and $16.0 \mathrm{~km} / \mathrm{s}$ in steps of 0.5 $\mathrm{km} / \mathrm{s}$. At each step within this speed range, the entry flight-path angle is varied in steps of $0.01 \mathrm{deg}$ between $-3.0 \mathrm{deg}$ and -10.0 $\mathrm{deg}$, so that the aerodynamic corridor ${ }^{15}$ can be defined. The target condition is to exit the atmosphere on an orbit with an apogee of $300 \mathrm{~km}$ in the same plane as the initial orbit.

For the purposes of this study, an "entry corridor" is defined as that region on the entry speed-entry flight-path angle plane within which the study configuration can achieve the target orbit subject to selected constraints. For example, if the vehicle can perform a circularization burn of $150 \mathrm{~m} / \mathrm{s}$ or less in $\Delta V$, then a contour can be drawn in the above plane within which the $\Delta V$ to circularize at 300 $\mathrm{km}$ would be less than or equal to this value. Regions in the plane outside this contour would violate the $\Delta V$ constraint and so would lie outside the entry corridor. Once the nominal entry speed for a mission is known and a nominal $\Delta V$ is defined for the vehicle, an entry corridor defined in this way would dictate the allowable range of entry flight-path angles.

The upper boundary of an entry corridor is defined by the shallowest fight-path angles at atmospheric entry for which successful aeropasses can be performed. Entries at shallower angles will result in skip-outs. An upper boundary tends to be rather "hard," especially at the higher entry speeds, in that changes in entry flight-path angle as small as 0.01 deg can mean the difference between achieving the desired orbit and remaining on an escape trajectory upon exit from the atmosphere. The lower boundary of the corridor is defined by the sleepest flight-path angles for which the target condition can be reached. Steeper entries will result in suborbital trajectories or excessively low orbits. A lower boundary tends to be much "softer" than an upper one, since small changes in entry flight-path angle result in exit orbits with similar parameters.

\section{Corridors When All Variations Active}

All modeled sources of variation and error were assumed to be active when performing the runs described below. For each run the atmosphere profile was randomly selected, as described earlier. Since it was assumed that the vehicle mass would be fairly well known, it was allowed to vary within a range of $\pm 1 \%$ from the nominal mass. The lift and drag coefficients varied in Gaussian fashion with distribution means centered at the nominal AFE values and standard deviations of 0.1 . The IMU error structure employed also reflected values characteristic of the AFE vehicle.

Figure 1 plots the mean plus 2-sigma values of $\Delta V$ to circularize at $300 \mathrm{~km}$ vs the entry flight-path angle for an entry speed of 12.0 $\mathrm{km} / \mathrm{s}$. For this cross section and for the complete entry corridor to be presented below, the GREGOAR bank margin was selected to be 45 deg. Fitted curves like that in Fig. 1 were used to determine 2sigma circularization $\Delta V$ contours on the entry corridor map. If one desired to specify a "nominal" circularization $\Delta V$ for an entry speed of $12.0 \mathrm{~km} / \mathrm{s}$, such a value could be found from Fig. 1 by selecting an acceptable entry flight-path angle and reading the corresponding $\Delta V$ off the curve.

Figure 2 presents the entry corridor map obtained using the fittedcurve method mentioned above. Circularization $\Delta V$ contours at $125 \mathrm{~m} / \mathrm{s}$ (darkest shading), $150 \mathrm{~m} / \mathrm{s}$ (medium shading), and $175 \mathrm{~m} / \mathrm{s}$ (lightest shading) are plotted within a wedge-shaped boundary that represents the study configuration capability unconstrained by vehicle limitations on heating rate, structural load, etc. These contours were determined from many thousands of cases where all sources of variation were allowed to vary.

Considering the $125-\mathrm{m} / \mathrm{s}$ zone, if a minimum 2-sigma corridor width of 0.5 deg is required to allow for navigation uncertainties, the highest speed for which the study configuration can be used is approximately $9.8 \mathrm{~km} / \mathrm{s}$. If this corridor width requirement is enforced, the study configuration is marginally suited to transfer missions from geosynchronous to low Earth orbit but is inadequate for higher energy missions. Considering the navigation infrastructure available in near-Earth space, a corridor width requirement of 0.5 deg is probably unnecessarily stringent. For entry at Mars such a requirement may be necessary.

If the $\Delta V$ budget is raised to $150 \mathrm{~m} / \mathrm{s}$, the entry speed that can be accommodated rises to at least $11.5 \mathrm{~km} / \mathrm{s}$. A bifurcation of the $150-\mathrm{m} / \mathrm{s}$ corridor occurs at entry speeds above this point. The upper branch is at first about $0.2 \mathrm{deg}$ in width, and the lower one is about $0.15 \mathrm{deg}$ in width. Thus neither branch meets the $0.5 \mathrm{deg}$ minimum width requirement.

If a 2-sigma $\Delta V$ budget of $175 \mathrm{~m} / \mathrm{s}$ is allowed, the study configuration can accommodate entry speeds up to approximately $13.7 \mathrm{~km} / \mathrm{s}$. The corridor bifurcates at slightly higher speeds. Shortly after the split, neither branch meets the minimum-width constraint, though both are wider than the branches in the previous case.

A further constraint that would quite likely be imposed on manned missions would be a maximum deceleration load limit. Previous studies ${ }^{16}$ have proposed a value of $5 g$ for such a limit. Application of this constraint imposes the most severe limits on the mission suitability of the study configuration, as can be seen in Fig. 3. If the 2-sigma $\Delta V$ limit is $175 \mathrm{~m} / \mathrm{s}$, the $0.5 \mathrm{deg}$ width requirement will make entry speeds above $12.5 \mathrm{~km} / \mathrm{s}$ unacceptable due to the deceleration loads encountered. Even if this width requirement is dropped, the 5 - $g$ constraint cuts off the 2-sigma corridor entirely by about $13.4 \mathrm{~km} / \mathrm{s}$. 


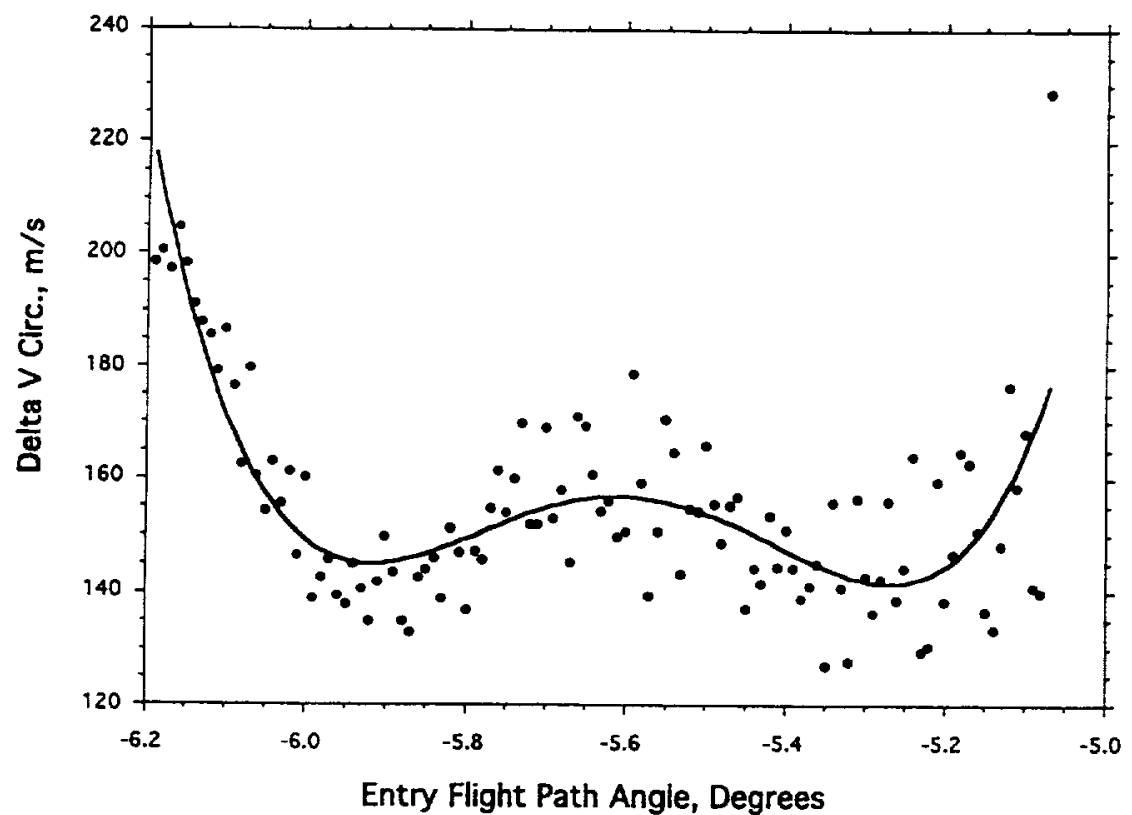

Fig. 1 Entry corridor cross section at $12.0 \mathrm{~km} / \mathrm{s}$.

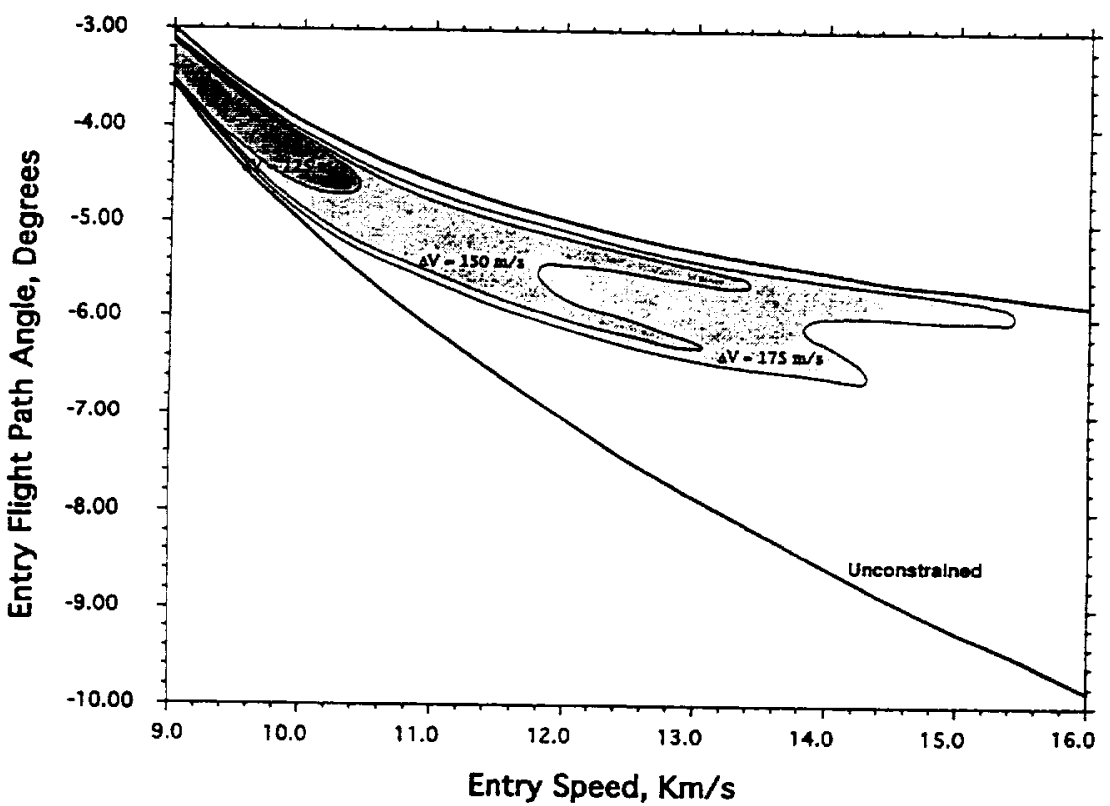

Fig. 2 Entry corridor for all variations active.

A similar corridor cutoff is seen in Braun et al..$^{5}$ at $13.9 \mathrm{~km} / \mathrm{s}$, but for a value of $L / D$ of 0.1 . For their case with an $L / D$ of 0.3 , which is similar to ours, the $g$-limited corridor extends well past $14.0 \mathrm{~km} / \mathrm{s}$. A point to consider in this comparison is that the corridors presented in our paper result from variational cases, with the boundaries determined by points 2 -sigma from the mean of the result; if only nominal or mean values of atmospheric density and vehicle parameters had been used, the corridors would have been wider. This seems to have been the case for the corridor determinations of Braun et al., which suggests one reason for their better corridor width results. When they performed an atmospheric dispersion analysis, they selected a nominal entry speed and flight-path angle combination (equivalent to selecting a "trough" point in Fig. 1) that resulted in a nominal value of circularization $\Delta V$ and then studied variations about this value. Their nominal $\Delta V$ value of $124.2 \mathrm{~m} / \mathrm{s}$ at an entry speed of 14 $\mathrm{km} / \mathrm{s}$ compares favorably with our 2 -sigma values at $12 \mathrm{~km} / \mathrm{s}$ seen in Fig. 1, which already incorporate dispersion effects

We must admit that the comparative simplicity of our algorithm may also have contributed to the narrowness of its corridor at higher speeds. A technique to mitigate the narrowing of the corridor due to an acceleration limit involves using bank angle modulation to provide load relief and so extend the flyable corridor to higher speeds. ${ }^{15}$ GREGOAR does not incorporate this strategy.

\section{Effect of Bank Margin on Entry Corridor}

One of the adjustable parameters of the GREGOAR guidance package is the bank margin. To examine the way in which changes in this parameter affect the entry corridor, two values besides the standard $45 \mathrm{deg}$ were tried, $30 \mathrm{deg}$ and $60 \mathrm{deg}$. A 2-sigma corridor cross section at $12.0 \mathrm{~km} / \mathrm{s}$ for all three bank margin numbers is presented in Fig. 4.

Recall that the bank margin is used to reduce the maximum lift the algorithm considers to be at its disposal when propagating the simulated trajectories prior to transition to the exit phase of flight. The larger the margin, the less lift considered, so that the transition will be commanded earlier. Consequently, penetration into the atmosphere will be shallower at the transition time, and speed at transition time will be greater. This results in greater propulsive braking requirements to achieve the target orbit. 


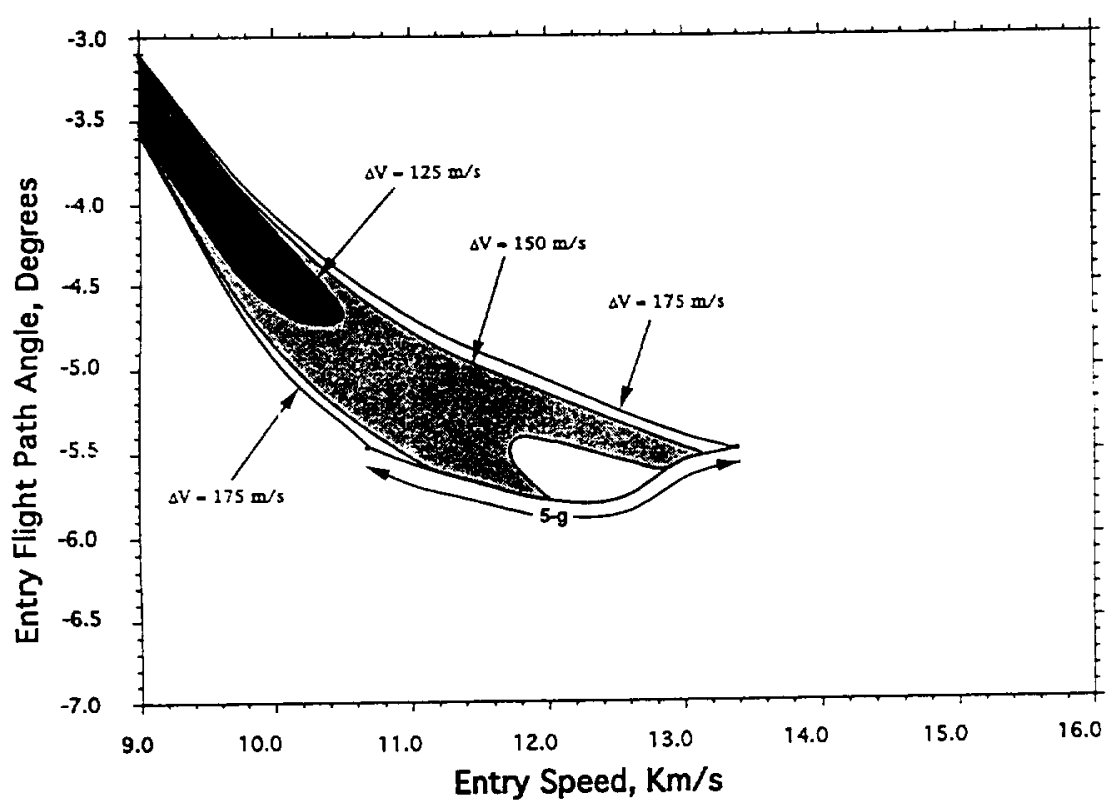

Fig. 3 Entry corridor limited by 5-g constraint.

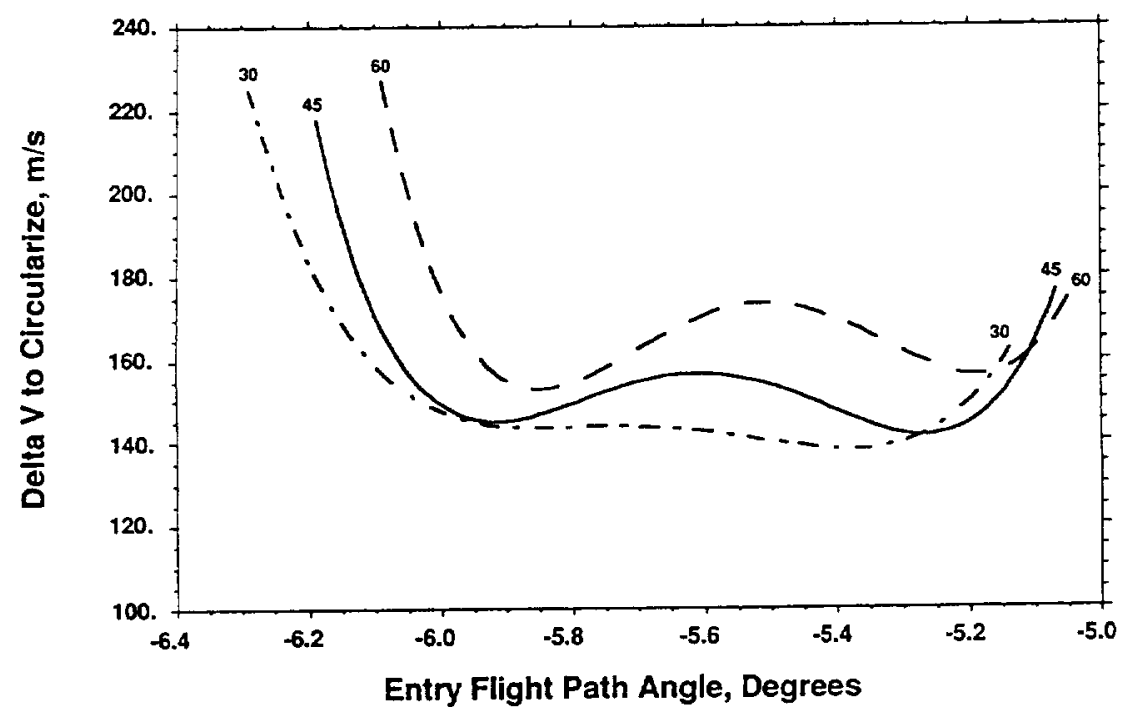

Fig. 4 Effect of bank margin on entry corridor cross section.

As can be seen in Fig. 4, the curve for 60 deg bank margin is shifted to higher circularization $\Delta V$ values when compared with that for 45 deg margin. Acceptable entry flight-path angles are also shallower for the $60 \mathrm{deg}$ margin setting. The corridor for this setting is somewhat narrower and barely avoids bifurcation for an allowed $\Delta V$ of $175 \mathrm{~m} / \mathrm{s}$. If only $150 \mathrm{~m} / \mathrm{s}$ is in the propulsion budget, the corridor is closed for this margin setting.

Considering a margin setting of $30 \mathrm{deg}$, fuller use of the vehicle's lift will be made by the algorithm, transition will be delayed, and more of the vehicle's velocity will be dissipated in the atmosphere. This can be seen in Fig. 4, where the curve for 30 deg margin reaches steeper entry angles, whereas minimum $\Delta V$ values are essentially the same as for the 45 deg setting. Note that the trendency to bifurcation of the corridor is greatly reduced for the $30 \mathrm{deg}$ value. This is important for extending the corridor to higher entry speeds. The $5-g$ deceleration constraint may be somewhat mitigated as well.

\section{Time Constant Effect on Corridor}

The effect of the other adjustable parameter in GREGOAR, the plane controller time constant, remains to be examined. Since we desire to improve the study configuration's performance, we select a bank margin setting of $30 \mathrm{deg}$, use values of 45 and $30 \mathrm{~s}$ for the time constant, and examine the results. Figure 5 shows a corridor cross section at $12.0 \mathrm{~km} / \mathrm{s}$ for the two time constant settings, where the corridor is expressed in terms of the wedge angle upon exit. The wedge angle combines the errors in inclination and right ascension of the node of the actual orbit with respect to the target orbit. In these simulations the target orbit plane was taken to be identical to the initial orbit plane, as stated earlier. In this figure the lower bounds of each zone represent mean values of the exit wedge angle resulting from their respective plane controller time constant values, whereas the upper bounds represent the mean plus 2-sigma exit wedge angle values.

Clearly, reducing the time constant reduces the plane error upon exit: The minimum wedge angle achieved for a time constant of 30 $s$ was $0.069 \mathrm{deg}$. whereas the minimum for $45 \mathrm{~s}$ was $0.171 \mathrm{deg}$. The spread in expected error is also reduced, as seen by the much smaller mean to 2-sigma band for the 30-s curves. This result confirms the expectations described at the end of the derivation section. As stated there, increased exit plane accuracy is purchased by an increase in the number of bank reversals during the aeropass. Recall that the bank reversals are not accomplished instantaneously, but are subject to a maximum rate of $20 \mathrm{deg} / \mathrm{s}$ and a maximum acceleration of $5 \mathrm{deg} / \mathrm{s}^{2}$. In selecting a time constant, therefore, consideration must 


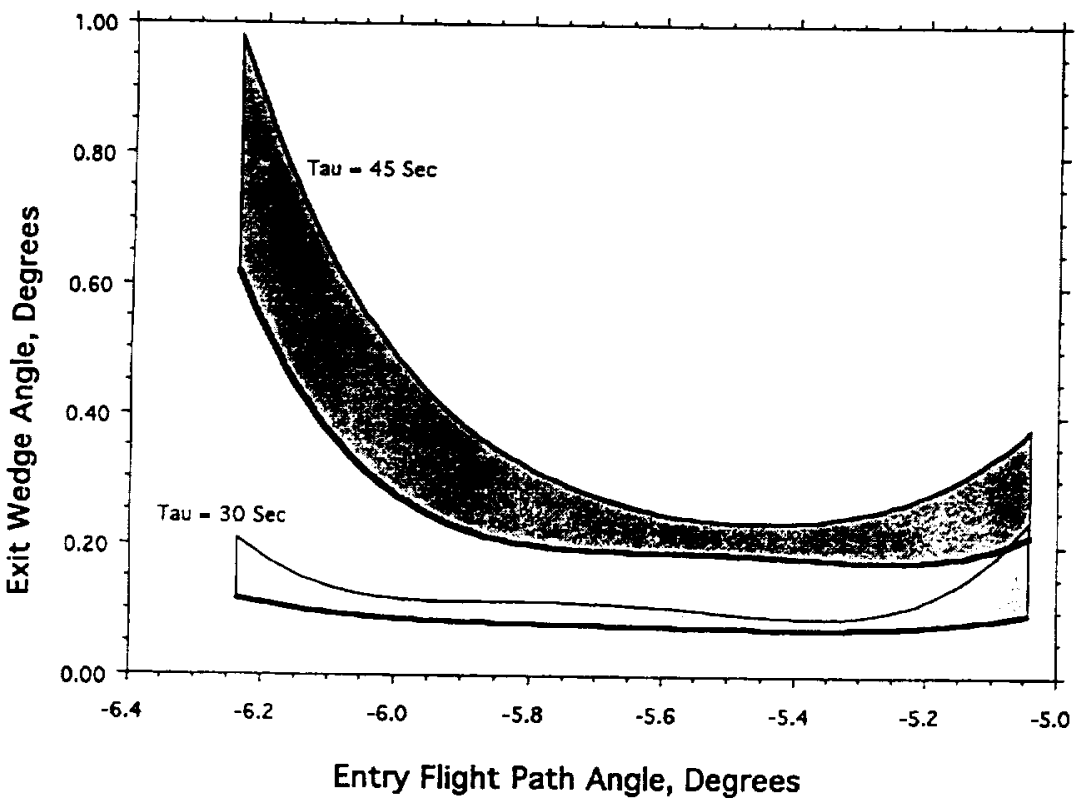

Fig. 5 Effect of different plane controller time constants.

be given to attitude control system requirements in performing bank reversals as well as the desired accuracy of the exit plane. Typically, four or five roll reversals are required with a time constant of $45 \mathrm{~s}$, whereas five or six roll reversals are required with a time constant of $30 \mathrm{~s}$. The number of required roll reversals can probably be reduced with slightly more sophisticated logic.

\section{Conclusions}

The GREGOAR algorithm has shown itself to be resilient to variations in fight parameters over a wide range of entry speeds. The tendency of the current version of this scheme to form bifurcated entry corridors could well limit its use on high-energy missions if large values of bank margin prove to be necessary. Further development work on the algorithm may reduce this characteristic splitting. In its current form the algorithm can be easily adapted for use in any number of aerobraking vehicles. Its minimum number of inputs and speed of execution, in addition to its robustness, recommend it highly for future use in aerobraking applications.

\section{Acknowledgment}

This work was performed as part of a Marshall Space Flight Center (NASA) Director's Discretionary Fund Project, no. 91-05, P05 (Aerocapture Tradeoff Analysis).

\section{References}

'Gamble, J. D., Cerimele, C. J., Moore, T. E., and Higgins, J., "Atmospheric Guidance Concepts for an Aeroassist Flight Experiment," Journal of the Astronautical Sciences, Vol. 36, Nos. 1/2, 1988, pp. 45-71

${ }^{2}$ Fuhry. D. P., "A Design Study of Onboard Navigation and Guidance During Aerocapture at Mars." Charles Stark Draper Labs., SDL-T-986, May 1988

${ }^{3}$ Shipley, B. W., and Ward, D. T., "Control Algorithms for Aerobraking in the Martian Atmosphere." American Astronomical Society. AAS $91-120$ Feb. 1990
${ }^{4}$ Willcockson, W. H., "Recent Developments in Aerocapture for the Mars Rover Sample Return Mission," American Astronomical Society, AAS 90012. Feb. 1990.

${ }^{5}$ Braun, R. D., Powell, R. W., and Lyne, J. E., "Earth Aerobraking Strategies for Manned Return from Mars," Journal of Spacecraft and Rockets, Vol 29, No. 3, 1992.

${ }^{6}$ Vinh, N. X., Optimal Trajectories in Atmospheric Flight, Elsevier, New York, 1981

${ }^{7}$ Vinh, N. X., Johannesen, J. R., Mease, K. D., and Hanson, J. M., "Explicit Guidance of Drag-Modulated Aeroassisted Transfer Between Elliptical Orbits," Journal of Guidance, Control, and Dynamics, Vol. 9, 1986. pp 274-280.

${ }^{8}$ Frostic, F., and Vinh, N. X., "Optimal Aerodynamic Control by Matched Asymptotic Expansions," Acta Astronautica, Vol. 3, pp. 319-332.

${ }^{9}$ Miele. A., and Venkataraman, P., "Optimal Trajectories for Aeroassisted Orbital Transfer," Acta Astronautica, Vol. 11, No. 7-8, 1984, pp 423-433.

${ }^{10}$ Miele, A., Basapur, V. K., and Mease, K. D., "Nearly-Grazing Optimal Trajectories for Aeroassisted Orbital Transfer," Journal of the Astronaurical Sciences, Vol. 34, No. 1, 1986, pp. 3-18.

${ }^{11}$ Miele, A., Basapur, V. K., and Lee, W. Y., "Optimal Trajectories for Aeroassisted, Noncoplanar Obital Transfer," Acta Astronautica, Vol. 15, Nos. 6/7, 1987, pp. 399-411.

${ }^{12}$ Miele, A., and Wang, T., "General Solution for the Optimal Trajectories of an AFE-Type Spacecraft," Aero-Astronautics, Rept. 254, Rice Univ. Houston, TX, 1991

13"Aeroassist Flight Experiment Preliminary Design Document," NASA Marshall Space Flight Center, Huntsville, AL, May 1986.

14 Justus, C. G." "The NASA/MSFC Global Reference Atmospheric Model-Mod 3," NASA Contractor Rept. 3256, 1980.

${ }^{15}$ Braun, R. D., and Powell, R. W., "Predictor-Corrector Guidance Algorithm for Use in High-Energy Aerobraking System Studies," Journal of Guidance, Control, and Dynamics, Vol. 15, No. 3, 1992, pp 672-678.

${ }^{16}$ Braun, R. D., and Powell, R. W., "Aerodynamic Requirements of a Manned Mars Aerobraking Transfer Vehicle," Journal of Spacecraft and Rockets, Vol. 28, No. 4, 1991, pp. 361-367. 